\title{
Characterization of soft x-ray echo-enabled harmonic generation free-electron laser pulses in the presence of incoherent electron beam energy modulations
}

\author{
N. S. Mirian $\odot,{ }^{1,2, *}$ G. Perosa $\odot,{ }^{2,3, \dagger}$ E. Hemsing, ${ }^{4}$ E. Allaria, ${ }^{1,2}$ L. Badano,${ }^{2}$ P. Cinquegrana, ${ }^{2}$ \\ M. B. Danailov, ${ }^{2}$ G. De Ninno, ${ }^{2}$ L. Giannessi, ${ }^{2,5}$ G. Penco, ${ }^{2}$ S. Spampinati, ${ }^{2}$ C. Spezzani, ${ }^{2}$ \\ E. Roussel, ${ }^{6}$ P. R. Ribič, ${ }^{2}$ M. Trovó, ${ }^{2}$ M. Veronese ${ }^{2},{ }^{2}$ and S. Di Mitri ${ }^{2,}{ }^{2}$ \\ ${ }^{1}$ Deutsches Elektronen-Synchrotron DESY, Notkestraße 85, 22607 Hamburg, Germany \\ ${ }^{2}$ Elettra-Sincrotrone Trieste S.C.p.A., 34149 Trieste, Italy \\ ${ }^{3}$ Università degli Studi di Trieste, Dipartimento di Fisica, Piazzale Europa 1, Trieste, Italy \\ ${ }^{4}$ SLAC National Accelerator Laboratory, Menlo Park, California 94025, USA \\ ${ }^{5}$ Istituto Nazionale di Fisica Nucleare-Laboratori Nazionali di Frascati (INFN), \\ 00044 Frascati, Rome, Italy \\ ${ }^{6}$ Université de Lille, CNRS, UMR 8523-PhLAM - Physique des Lasers Atomes et Molécules \\ F-59000 Lille, France
}

(Received 7 June 2021; accepted 30 July 2021; published 20 August 2021)

\begin{abstract}
Echo-enabled harmonic generation free-electron lasers (EEHG FELs) are promising candidates to produce fully coherent soft $\mathrm{x}$-ray pulses by virtue of efficient high-harmonic frequency up-conversion from ultraviolet lasers. The ultimate spectral limit of EEHG, however, remains unclear, because of the broadening and distortions induced in the output spectrum by residual broadband energy modulations in the electron beam. We present a mathematical description of the impact of incoherent (broadband) energy modulations on the bunching spectrum produced by the microbunching instability through both the accelerator and the EEHG line. The model is in agreement with a systematic experimental characterization of the FERMI EEHG FEL in the photon energy range 130-210 eV. We find that amplification of electron beam energy distortions primarily in the EEHG dispersive sections explains an observed reduction of the FEL spectral brightness proportional to the EEHG harmonic number. Local maxima of the FEL spectral brightness and of the spectral stability are found for a suitable balance of the dispersive sections' strength and the first seed laser pulse energy. Such characterization provides a benchmark for user experiments and future EEHG implementations designed to reach shorter wavelengths.
\end{abstract}

DOI: $10.1103 /$ PhysRevAccelBeams.24.080702

\section{INTRODUCTION}

Free electron lasers (FELs) have enabled a new way for researchers to explore electronic dynamics at molecular and atomic scales via femtosecond pulses, gigawatt peak powers, and tunable wavelengths in the range of extreme ultraviolet to hard $\mathrm{x}$ rays [1]. Self-amplified spontaneous emission FELs generate a spiky spectrum and therefore offer relatively poor longitudinal coherence $[2,3]$. Seeded FELs are, at present, the only devices producing stable

najmeh.mirian@desy.de

giovanni.perosa@elettra.eu

simone.dimitri@elettra.eu

Published by the American Physical Society under the terms of the Creative Commons Attribution 4.0 International license. Further distribution of this work must maintain attribution to the author(s) and the published article's title, journal citation, and DOI. pulses with good longitudinal coherence at wavelengths now approaching the water window $[4,5]$.

Echo-enabled harmonic generation (EEHG) was conceived as a seeding method with excellent high-harmonic conversion efficiency to generate transform-limited radiation pulses down to soft $\mathrm{x}$ rays [6-11]. By utilizing two laser modulations and dispersive sections (DSs), a monochromatic (coherent) energy modulation is imprinted on to the relativistic electron beam and transformed to a highharmonic density modulation (see Fig. 1). The beam then enters the undulator radiator where the density-modulated (bunched) electrons radiate coherently at wavelengths up to $\sim 100$ times shorter than that of the ultraviolet (UV) seeding lasers. With sufficient gain, the radiation can be amplified up to saturation.

Recently, the impact of phase variations in the seed lasers on EEHG performance was investigated experimentally [12], illustrating the capability to shape the EEHG FEL spectrum by tuning the second seed laser power and phase. The agreement between experimental data and the 

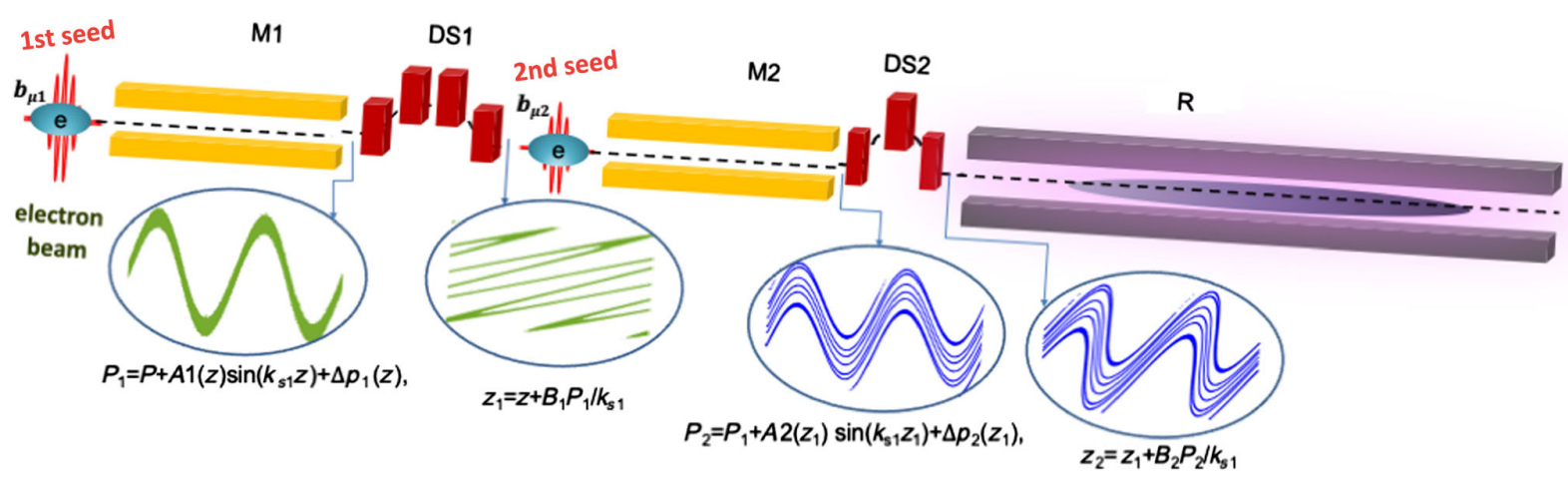

FIG. 1. Main components of the EEHG scheme: first modulator (M1), strong first dispersive section (DS1), second modulator (M2), and weaker second dispersive section (DS2). Equations refer to quantities of the electron beam longitudinal phase space introduced in Eq. (1). In particular, $\Delta p_{1,2}$ is the incoherent energy modulation [see Eq. (2)] and $b_{\mu 1, \mu 2}$ is the incoherent bunching factor [see Eq. (13)]. After DS2, the nano-bunched electron beam travels into the radiator (R) and emits coherent and powerful light pulse.

preceding theory [13-15] was obtained in a condition in which energy nonuniformities of the electron beam could be neglected. On the other hand, it is well known that energy distortions in the electron beam can impact the EEHG bunching spectrum (see, e.g., Refs. [16-18]). Particularly relevant to this work, the impact of incoherent energy modulations on EEHG performance was discussed theoretically in Refs. [19-21]. Here, we examine the details of these studies by means of a extensive theoretical formulation of the evolution of energy and density modulations in EEHG. In particular, we examine and compare the measured FEL performance with an analytical model that includes incoherent modulations in the electron beam longitudinal phase space that develop from the early beam acceleration process through the final EEHG transformations.

It is known that in seeded FEL systems, uncontrolled energy structures can lead to a broader FEL bandwidth and reduced peak spectral intensity. These energy structures, accumulated during beam manipulation in the accelerator, can hardly be removed completely. They can introduce extra frequencies into the FEL gain bandwidth that deteriorate the longitudinal coherence promised by external seeding [21-25]. Such structures are generally due to the buildup of beam collective effects such as coherent synchrotron radiation (CSR) and longitudinal space charge (LSC) during acceleration and compression, both contributing to the socalled microbunching instability (MBI) [26-28] whose maximum gain, depending on the compression setting, is typically at final wavelengths $\lambda_{0} \gtrsim 0.5 \mu \mathrm{m}$. Here, we report on results of a systematic investigation at the FERMI FEL operating in EEHG mode [10] where we find good agreement between theory and experimental data. These studies help to benchmark the analytic model which thus provides a practical tool for the design and optimization of EEHG sources at even shorter wavelengths.

The paper is organized as follows. Section II discusses the theory of MBI in EEHG. Section III presents the experiment results and compares them with theory. Conclusions are reached in Sec. IV.

\section{COHERENT AND INCOHERENT ENERGY AND DENSITY MODULATIONS}

\section{A. Theoretical background}

The evolution of the electron beam longitudinal phase space through the EEHG line in the presence of energy distortions is described by the following equations $[20,21]$ :

$$
\begin{aligned}
P_{1} & =P+A_{1}(z) \sin \left(k_{s 1} z\right)+\Delta p_{1}(z), \\
z_{1} & =z+B_{1} P_{1} / k_{s 1}, \\
P_{2} & =P_{1}+A_{2}\left(z_{1}\right) \sin \left(k_{s 2} z_{1}\right)+\Delta p_{2}\left(z_{1}\right), \\
z_{2} & =z_{1}+B_{2} P_{2} / k_{s 1},
\end{aligned}
$$

where $A_{1,2}(z)=\Delta E_{1,2}(z) / \sigma_{E}$ is the normalized coherent energy modulation from seed lasers, $B_{1,2}=k_{s 1} R_{56}^{(1,2)} \sigma_{E} / E$ is the normalized energy dispersion in the chicanes, $E$ is the electron beam mean energy, $\sigma_{E}$ is the rms slice energy spread, and $\Delta p_{1,2}(z)$ is the energy distortions of the electron beam distribution. The role of these terms is carefully investigated in the following.

In this description, $\Delta p_{1}$ represents any energy structure accumulated in the electron beam up to the entrance of the first EEHG chicane $B_{1} . \Delta p_{2}$ is used to capture the integrated effect of CSR from $B_{1}$ and of LSC in second modulator. They can be expressed as the superposition of monochromatic modulations of different amplitudes [21,29],

$$
\Delta p_{1,2}(z)=\sum_{\mu=0}^{\infty} p_{1,2}\left(k_{\mu}\right) \sin \left(k_{\mu} z+\phi_{1 \mu, 2 \mu}\right)
$$

where $\phi_{1,2 \mu}$ is a random phase.

When the energy distortions $\Delta p_{1,2}$ are ignored and in the assumption of uniform lasers $A_{1,2}(z)=A_{1,2}$ (i.e., seed 
durations much longer than bunch duration), the Fourier transform of the electron beam density distribution-the so-called bunching factor-can be calculated at the exit of the second EEHG modulator according to [6],

$$
\bar{b}_{n, m}\left(k_{E}\right)=e^{-\zeta_{E}^{2} / 2} J_{n}\left(-\zeta_{E} A_{1}\right) J_{m}\left(-a_{E} A_{2} B_{2}\right),
$$

where $a_{E}=n+m k_{s 2} / k_{s 1}$ is the harmonic number with integer numbers $n$ and $m$. The EEHG wave number is $k_{E}=a_{E} k_{s 1}$ and $\zeta_{E}=n B_{1}+a_{E} B_{2}$. This factor is known to help characterize the EEHG performance and is optimized approximately at $\zeta_{E}=j_{n, 1}^{\prime} / A_{1}$ where $j_{n, 1}^{\prime}$ is the first root of $J_{n}^{\prime}$.

If the energy distortion $\Delta p_{2}$ is sufficiently slowly varying longitudinally, i.e., $k_{\mu} B_{1} A_{1} / k_{1} \ll 2 \pi$, that we can approximate its functional dependence as $z_{1} \approx z$. This approximation $\Delta p_{2}\left(z_{1}\right)=\Delta p_{2}(z)$ is reasonable when the wavelengths associated with $\Delta p_{2}$ are long enough that sampling at the electron position $z$ is a good approximation to sampling at the shifted position $z_{1}$. Then the EEHG bunching factor close to the harmonic peak becomes $[15,21]$

$$
\begin{aligned}
b_{n, m}(k)= & e^{-\frac{1}{2}\left(\zeta_{E}+\frac{k-k_{E}}{k_{1 s}} B\right)^{2}} \int_{-\infty}^{+\infty} d z f(z) J_{m}\left[-\frac{k}{k_{s 1}} B_{2} A_{2}(z)\right] \\
& \times J_{n}\left[-\left(\zeta_{E}+\frac{k-k_{E}}{k_{s 1}} B\right) A_{1}(z)\right] \\
& \times e^{i\left(-\zeta_{E} \Delta p_{1}(z)-a_{E} B_{2} \Delta p_{2}(z)+\left(k-k_{E}\right) z\right)}
\end{aligned}
$$

where $f(z)$ is electron beam density distribution function and $B=B_{1}+B_{2}$. In the first two gain lengths in the radiator ( $\mathrm{R}$ in Fig. 1), the intensity of the FEL radiation is estimated to grow like $\propto z^{2}\left|b_{n, m}(k)\right|^{2}$. In the limit of negligible slippage, the final radiation spectral pulse properties are given by $\left|b_{n, m}(k)\right|^{2}$. Thus, by virtue of the general expression for the energy modulations given in Eq. (2), this equation can be used to quantify the spectral effect of broadband energy modulations induced by MBI on the FEL output.

\section{B. Bunching phase}

The $z$-dependent additional bunching phase due to electron beam energy distortions in Eq. (4) is

$$
\psi(z)=-\zeta_{E} \Delta p_{1}(z)-a_{E} B_{2} \Delta p_{2}(z) .
$$

From this, one can obtain the moments of the spectral bunching distribution and gain insight into the relative magnitude of the contributions from $\Delta p_{1,2}$ [21].

MBI-induced energy modulations accumulated up to the exit of the first modulator, $\Delta p_{1}$, are multiplied by the small scaling parameter $\left|\zeta_{E}\right| \lesssim 1$. Linear, quadratic, and sinusoidally shaped initial modulations were investigated in Ref. [20], where it was shown that the smallness of $\zeta_{E}$ accounts for the insensitivity of the EEHG bunching spectrum to small initial perturbations. However, as discussed in Ref. [21], energy modulations $\Delta p_{2}$ that develop between the EEHG chicanes are multiplied by the much larger factor $a_{E} B_{2} \approx m / A_{2} \gg 1$, and therefore can have a noticeable impact on the final bunching spectrum at high harmonics.

The rms bandwidth of $\left|b_{n, m}(k)\right|^{2}$ in Eq. (4) is $\sigma_{k}^{2}=$ $\sigma_{k s}^{2}+\sigma_{\psi^{\prime}}^{2}$, where $\sigma_{k s}$ is the transform-limited (TL) bandwidth and $\sigma_{\psi^{\prime}}^{2}=\left\langle\left[\psi^{\prime}-\left\langle\psi^{\prime}\right\rangle\right]^{2}\right\rangle$ is the bandwidth due to the nonlinear phase structure, where brackets denote integration over the $z$-dependent amplitudes in the integrand in Eq. (4). Assuming the bunching longitudinal envelope is determined by the second seed laser and that it is a TL Gaussian pulse, the relative bandwidth in the case of optimized bunching absent MBI can be approximated as [15]

$$
\bar{\sigma}_{k s}^{2}=\frac{4 \bar{\sigma}_{k s 2}^{2}}{3 m^{4 / 3}},
$$

where $\bar{\sigma}_{k s 2}$ is the relative bandwidth of the second seed laser.

Inserting Eq. (2) for broadband energy distortions into the phase in Eq. (5), the instantaneous spatial bunching frequency is $k_{z}=k_{E}+\psi^{\prime}(z)$, where

$$
\begin{aligned}
\psi^{\prime}(z)= & -\zeta_{E} \sum_{\mu=0}^{\infty} p_{1}\left(k_{\mu}\right) k_{\mu} \cos \left(k_{\mu} z+\phi_{1 \mu}\right) \\
& -a_{E} B_{2} \sum_{\mu=0}^{\infty} p_{2}\left(k_{\mu}\right) k_{\mu} \cos \left(k_{\mu} z+\phi_{2 \mu}\right)
\end{aligned}
$$

is the $z$-derivative of the additional phase. The mean bunching frequency is then $\left\langle k_{z}\right\rangle$. Thus, $\left\langle\psi^{\prime}\right\rangle$ gives the spectral shift from $k_{E}$, and $\sigma_{\psi^{\prime}}$ gives the excess bandwidth due to the distortions. Assuming that the characteristic MBI wavelengths are small compared to the length of the bunching envelope (e.g., $k_{\mu} \gg \sigma_{k s}$ ) and that the individual phases $\phi_{1,2 \mu}$ are uncorrelated over $\mu$, bandwidth of $\left|b_{n, m}(k)\right|^{2}$ is therefore

$$
\sigma_{k}^{2}=\sigma_{k s}^{2}+\sum_{\mu=0}^{\infty}\left[\frac{\zeta_{E}^{2}}{2}\left(p_{1}\left(k_{\mu}\right) k_{\mu}\right)^{2}+\frac{\left(a_{E} B_{2}\right)^{2}}{2}\left(p_{2}\left(k_{\mu}\right) k_{\mu}\right)^{2}\right] .
$$

\section{Bunching amplitude}

Similarly to the phase, the bunching factor in Eq. (4) for generic energy distortions is here specialized for MBI-induced energy modulations described in Eq. (2). It becomes 


$$
\begin{aligned}
b_{n, m}(k)= & \int \chi(z, k) e^{-i\left(z\left(k-k_{E}\right)+\psi(z)\right)} d z \\
= & \int d z \chi(z, k) \prod_{\mu=0}^{\infty} \sum_{l_{1}=-\infty}^{\infty} \sum_{l_{2}=-\infty}^{\infty} J_{l_{1}}\left(-\zeta_{E} p_{1}\left(k_{\mu}\right)\right) \\
& \times J_{l_{2}}\left(-a_{E} B_{2} p_{2}\left(k_{\mu}\right)\right) \\
& \times e^{-i z\left[k_{\mu}\left(l_{1}+l_{2}\right)-\left(k-a_{E} k_{1 s}\right)\right]} e^{-i\left(l_{1} \phi_{1 \mu}+l_{2} \phi_{2 \mu}\right)}
\end{aligned}
$$

where

$$
\begin{aligned}
\chi(z, k)= & e^{-\frac{1}{2}\left(\zeta_{E}+\frac{k-k_{E}}{k_{1 s}} B\right)^{2}} J_{m}\left[-\frac{k}{k_{s 1}} B_{2} A_{2}(z)\right] \\
& \times J_{n}\left[-\left(\zeta_{E}+\frac{k-k_{E}}{k_{s 1}} B\right) A_{1}(z)\right] f(z) .
\end{aligned}
$$

With the definition of the bunching spectrum $b_{n, m}(k)$, we can now quantify the presence of sidebands and/or of a broader spectral pedestal in EEHG. The EEHG bunching amplitude evaluated for $k=a_{E} k_{1 s}$ can be calculated when $l_{1}=-l_{2}$, so that

$$
\begin{aligned}
b_{n, m}\left(a_{E} k_{1 s}\right)= & \grave{b}_{n, m} \prod_{\mu=0}^{\infty} \sum_{l_{1}=-\infty}^{\infty}(-1)^{l_{1}} J_{l_{1}}\left(-\zeta_{E} p_{1}\left(k_{\mu}\right)\right) \\
& \times J_{l_{1}}\left(-a_{E} B_{2} p_{2}\left(k_{\mu}\right)\right) e^{-i l_{1}\left(\phi_{1 \mu}-\phi_{2 \mu}\right)}
\end{aligned}
$$

where $\grave{b}_{n, m}$ is the $z$-integration of Eq. (10) for $k=a_{E} k_{1 s}$ and demonstrates the bunching factor when MBI is absent. In above equation, we use the Bessel function relationship for integer $\nu$ value $J_{-\nu}(x)=(-1)^{\nu} J_{\nu}(x)$. In the case of long seed lasers (i.e., $\left.A_{1,2}(z)=A_{1,2}\right)$ and a uniform electron beam, it is easy to see that $\grave{b}_{n, m}=\bar{b}_{n, m}$. Note that the bunching is suppressed at the roots of the two Bessel functions. Assuming that the arguments of $J_{l_{1}}$ in Eq. (11) are less than 1, the high order of Bessel functions can be ignored and the leading term can be expanded around 0 . In doing so, the bunching factor can be simplified to

$$
\begin{aligned}
& b_{n, m}\left(a_{E} k_{1 s}\right) \\
& \approx \grave{b}_{n, m} \prod_{\mu=0}^{\infty} J_{0}\left(-\zeta_{E} p_{1}\left(k_{\mu}\right)\right) J_{0}\left(-a_{E} B_{2} p_{2}\left(k_{\mu}\right)\right) \\
& \approx \grave{b}_{n, m} \prod_{\mu=0}^{\infty}\left[1-\frac{1}{4}\left(\left(\zeta_{E} p_{1}\left(k_{\mu}\right)\right)^{2}+\left(a_{E} B_{2} p_{2}\left(k_{\mu}\right)\right)^{2}\right)\right] .
\end{aligned}
$$

\section{Modeling the microbunching instability}

For numerical modeling, the expressions of the perturbed bunching factor can now be made explicit for the MBIinduced broadband energy modulations accumulated up to the second EEHG DS. At typical frequencies $k_{\mu} \gg 1 / \sigma_{z}$, we can write $[21,30]$

$$
p_{1,2}\left(k_{\mu}\right)=4 \pi b_{\mu 1, \mu 2}\left(k_{\mu}\right) \frac{I}{I_{A}} \frac{Z_{1,2}\left(k_{\mu}\right)}{Z_{0} \sigma_{\gamma}} L_{1,2}
$$

where $p_{1,2}, b_{\mu 1, \mu 2}\left(k_{\mu}\right)$, and $Z_{1,2}(k)$ are the broadband energy modulation, broadband bunching factor, and LSC impedance per unit length in the first and second modulator of length $L_{1,2} . I$ and $I_{A}=17045 \mathrm{~A}$ are the bunch peak current and the Alfven current, and $Z_{0}=377 \Omega$ is the free space impedance. The in-vacuum LSC impedance $Z\left(k_{\mu}\right)$ through the modulator is [30,31]

$$
Z\left(k_{\mu} / C\right)=\frac{i Z_{0} k_{\mu}}{4 \pi \gamma_{z}^{2} C}\left[1+2 \ln \left(\frac{\gamma_{z}}{k_{\mu} r_{b}}\right)\right],
$$

with the effective beam transverse size $r_{b}=0.8735 \sqrt{\epsilon_{x} \beta_{x}+\epsilon_{y} \beta_{y}}$, where $\epsilon_{x, y}$ and $\beta_{x, y}$ are emittance betatron functions in $x$ and $y$ directions, $\gamma_{z}=$ $\gamma / \sqrt{1+K_{u}^{2} / 2}$ is the longitudinal Lorentz factor inside an undulator with the (peak) untapered undulator parameter $K_{u}$, and $C$ is compression factor.

The MBI-induced energy modulation $\Delta p_{1,2}$ in Eq. (2) is calculated numerically by means of a comprehensive linear gain model of the instability from beam injection into the accelerator up to the undulator line, including longitudinal energy-dispersion function $\left(R_{56}\right)$ and CSR in the magnetic compressor, LSC and intrabeam scattering, and beam heating at low energy [32,33]. The model starts from a shot noise like initial bunching factor and provides the bunching factor at any point along the line as well.

In the simplified case of single-stage beam compression and assuming linear gain regime of the instability, the amplification of density modulation, or gain [26,28], in the presence of an arbitrary incoming energy distribution $V\left(P_{0}\right)[31]$, goes like

$$
\begin{aligned}
G=\left|\frac{b_{\mu f}}{b_{\mu 0}}\right| \simeq & \frac{I}{\gamma I_{A}}\left|k_{f} R_{56} \int_{0}^{L} d s \frac{4 \pi Z\left(k_{\mu 0}, s\right)}{Z_{0}}\right| \\
& \times \int d P_{0} V\left(P_{0}\right) e^{-i k_{\mu f} R_{56} P_{0}},
\end{aligned}
$$

where $k_{\mu f}=k_{\mu 0} /\left(1+h R_{56}\right), k_{\mu 0}$ and $k_{\mu f}$ are modulation wavelength before and after the beam compression, and $h$ is the initial beam energy chirp.

Equation (15) suggests that the gain can be reduced by energy Landau damping, i.e., by increasing the beam uncorrelated energy spread at relatively low-beam energies. Indeed, this is now accomplished at several FEL facilities through the laser heater (LH) system [30,34-36], whose accurate control has become a tool to optimize the FEL spectral brightness in the presence of MBI. 


\section{EEHG MEASUREMENTS}

\section{A. Bandwidth enlargement and central frequency fluctuation}

The EEHG experiment was conducted with an electron beam accelerated through the FERMI linac to the final energy of $E=1.32 \mathrm{GeV}$. The beam normalized emittance measured in front of the undulator amounts to approximately $1 \mathrm{~mm}$ mrad in both transverse planes. The electron bunch is compressed by a factor $C \sim 10$ to reach a final peak current in the core of $I=700$ A.

In Fig. 2, the left plot shows the rms spectral bandwidth of the FEL at harmonic $a_{E}=30$ of a UV seed laser $\left(\lambda_{s}=264.54 \mathrm{~nm}\right)$, as a function of the LH-induced energy spread. The dispersion of the first EEHG DS was set to $R_{56}^{(1)}=2.25 \mathrm{~mm}$. The blue curve shows the EEHG emission for $n=-1$ configuration or $R_{56}^{(2)}=75 \mu \mathrm{m}$ and the red curve is for $n=-2$ or $R_{56}^{(2)}=145 \mu \mathrm{m}$ [ $n$ defined in Eq. (3)]. The error bars reflect the rms fluctuation of experimental data collected over a series of 20 consecutive shots at $10 \mathrm{~Hz}$ machine repetition rate. The experimental data are compared with the theoretical bandwidth predicted by Eq. (8) for $n=-1$ and $n=-2$, illustrated by the dashed-dotted blue and red line, respectively. For comparison, the green dashed line represents the bandwidth for optimized bunching absent MBI, Eq. (6), assuming TL seed laser pulses with a FWHM bandwidths of $2.01 \mathrm{~nm}$ and $1.35 \mathrm{~nm}$, respectively.

The spectra of the energy distortion amplitudes predicted by the MBI model for two different LH energy spread settings is shown in the right Fig. 2 subplots. The integrated impact of these distortions matches well with the measured
FEL bandwidth, which is substantially reduced for a LHinduced energy spread $\geq 30 \mathrm{keV}$. The model allows us to explain the observations on the basis of MBI-induced energy modulations augmented by the first EEHG DS, where $p_{2}\left(\lambda_{\mu}\right)$ in absence of the first seed results always larger than $p_{1}\left(\lambda_{\mu}\right)$.

The different MBI sensitivity of the EEHG bandwidth for the cases $n=-1$ and $n=-2$ is explained by means of Eq. (8). On the one side, $p_{1}\left(\lambda_{\mu}\right)$ is multiplied by the EEHG scaling factor, which therefore can be modified to change the sensitivity of the final bunching to the electron beam energy perturbations coming from the accelerator. On the other side, $p_{2}\left(\lambda_{\mu}\right)$ is multiplied by $a_{E} B_{2}$, with $\left|B_{2}\right| \approx n B_{1} / a_{E}$, such that a higher value of $|n|$ forces larger values $R_{56}^{(2)}$ of the second dispersion section.

Equation (7) suggests that, by virtue of larger values of $B_{2}$ in the presence of MBI, frequency fluctuations in the configuration $n=-2$ for fixed $\zeta_{E}$, should be larger when $n=-1$. Figure 3 compares the range of the frequency fluctuation by showing the standard deviation of 50 single shots of $n=-1$ (blue) and 100 shots of $n=-2$ (red) configurations in EEHG experiment at $\lambda_{\mathrm{FEL}}=8.8 \mathrm{~nm}$ respect to the different induced LH energy spread. In other words, this figure shows the range of $\left\langle k_{z}\right\rangle$ fluctuations for different level of incoherent energy modulation. The larger fluctuations seen with the $n=-2$ setting align with expectations.

\section{B. Pulse intensity reduction}

In Fig. 4, the top plot shows the maximum measured FEL intensity for $n=-1$ (blue line) and $n=-2$ (red line). As mentioned the FEL intensity scales with $\left|b_{n, m}\left(k_{E}\right)\right|^{2}$.
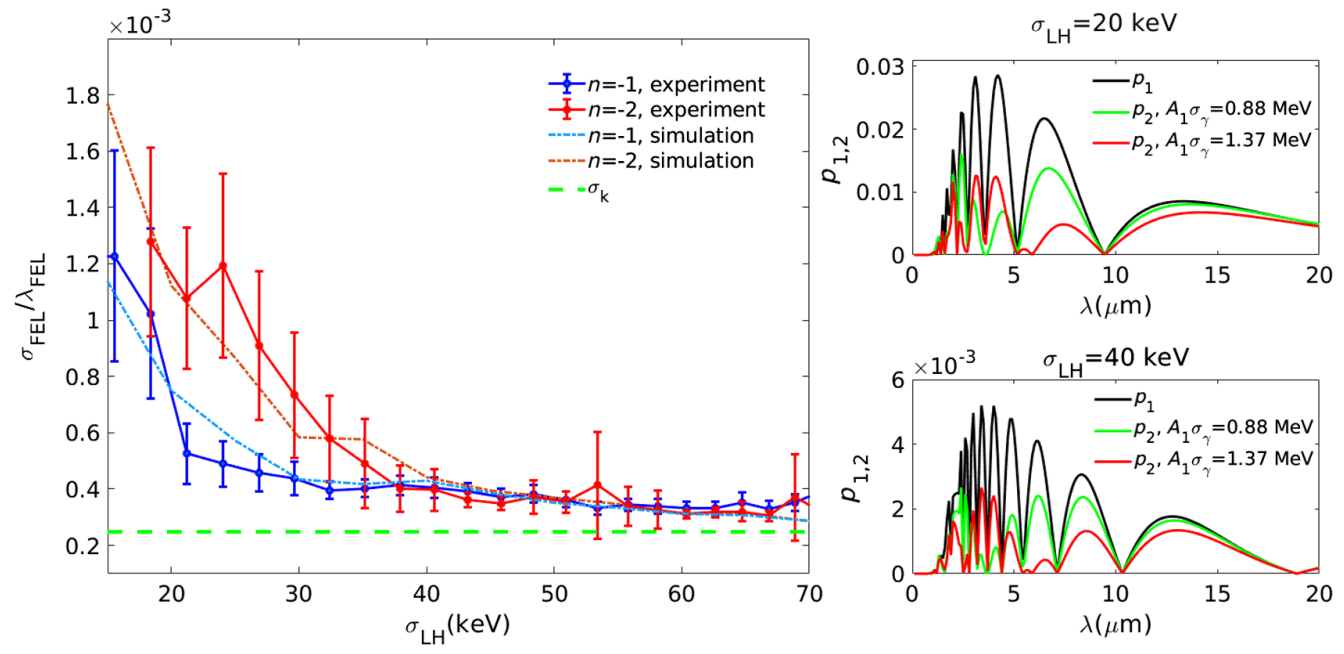

FIG. 2. Left: Relative FEL rms spectral bandwidth vs LH-induced energy spread. Blue and red lines are experimental data (solid) and theoretical prediction [dashed-dotted, Eq. (8)] for $n=-1$ and $n=-2$, respectively. EEHG is tuned at $\lambda_{\mathrm{FEL}}=8.8 \mathrm{~nm}$. The dashed green line is from Eq. (6). In case of $n=-1$, the first seed energy is $8.3 \mu \mathrm{J}\left(A_{1} \sigma_{E}=0.88 \mathrm{MeV}\right)$ and for $n=-2$, it is $20 \mu \mathrm{J}$ $\left(A_{1} \sigma_{E}=1.37 \mathrm{MeV}\right)$. Right: calculated energy modulation amplitude $p_{1}\left(\lambda_{\mu}\right)$ and $p_{2}\left(\lambda_{\mu}\right)$ from MBI modeling for $\sigma_{\mathrm{LH}}=20 \mathrm{keV}$ (top) and $40 \mathrm{keV}$ (bottom). 


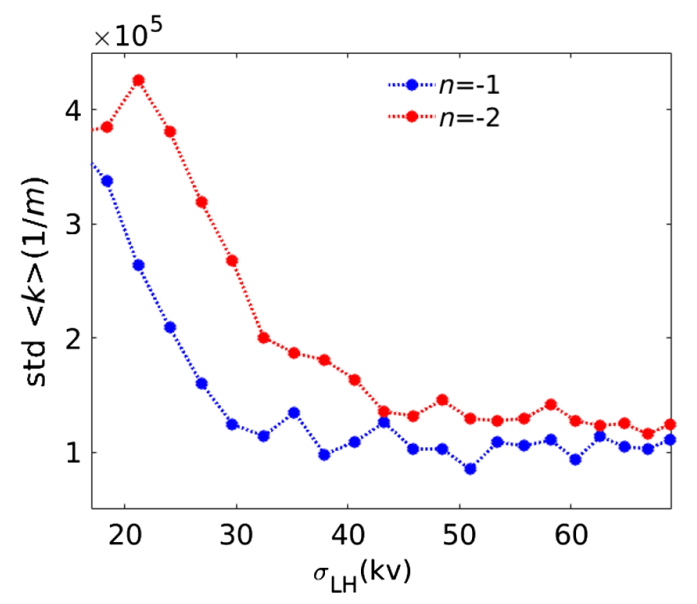

FIG. 3. Comparison of standard deviation of frequency fluctuations of 50 shots $n=-1$ (blue) and 100 shots $n=-2$ (red) configurations in EEHG experiment respect to the different induced LH energy spread. EEHG harmonic is $30\left(\lambda_{\mathrm{FEL}}=\right.$ $8.8 \mathrm{~nm}$ ). The FEL parameters are same as in Fig. 2.

The bottom plot shows the values calculated from Eq. (12). The equation shows that when the MBI gain is suppressed by high-LH pulse energies, the product function $\quad \Gamma=\prod_{\mu=0}^{\infty} J_{0}\left(-\zeta_{E} p_{1}\left(k_{\mu}\right)\right) J_{0}\left(-a_{E} B_{2} p_{2}\left(k_{\mu}\right)\right) \approx$ $\prod_{\mu=0}^{\infty}\left[1-\frac{1}{4}\left(\left(\zeta_{E} p_{1}\left(k_{\mu}\right)\right)^{2}+\left(a_{E} B_{2} p_{2}\left(k_{\mu}\right)\right)^{2}\right)\right]$ tends to 1 . Likewise, when the MBI is more pronounced at low-LH pulse energies, the product function approaches zero. At the same time, owing to the large LH-induced energy spread $(>40 \mathrm{keV})$, the FEL gain is diminished and therefore the FEL intensity is reduced. We note that in the $\Gamma$ function, $p_{2}\left(\lambda_{\mu}\right)$ is multiplied by $a_{E} B_{2}$ which explains the different behavior of the function for $n=-1$ and $n=-2$, in agreement with the experimental observation.

\section{Impact of first seed laser}

It is well known that in the processes of harmonic emission driven by an external laser, the seed laser-induced energy modulation has to exceed the uncorrelated energy spread of the beam at the undulator entrance. Moreover, the EEHG bunching becomes less sensitive to MBI with increased laser modulations. This leads to the question if and to which extent the seeding laser pulse energy could be increased in order to counteract the effect of MBI, before preventing any further lasing by exceeding the FEL normalized energy bandwidth.

To answer this question, In Fig. 5, the left plot illustrates the FEL intensity recorded as function of the first seed laser pulse energy, for two values of the LH pulse energy. Since the bunching is more sensitive to the coherent energy modulation in the first modulator at higher harmonics, the experiment was done for harmonic 18 . The beam energy was $1.1 \mathrm{GeV}$ and the compression factor about 7 , for approximately $550 \mathrm{~A}$ in the bunch core. EEHG was set in configuration $n=-1\left(R_{56}^{(1)}=1.9 \mathrm{~mm}\right.$ and $\left.R_{56}^{(2)}=98 \mu \mathrm{m}\right)$.
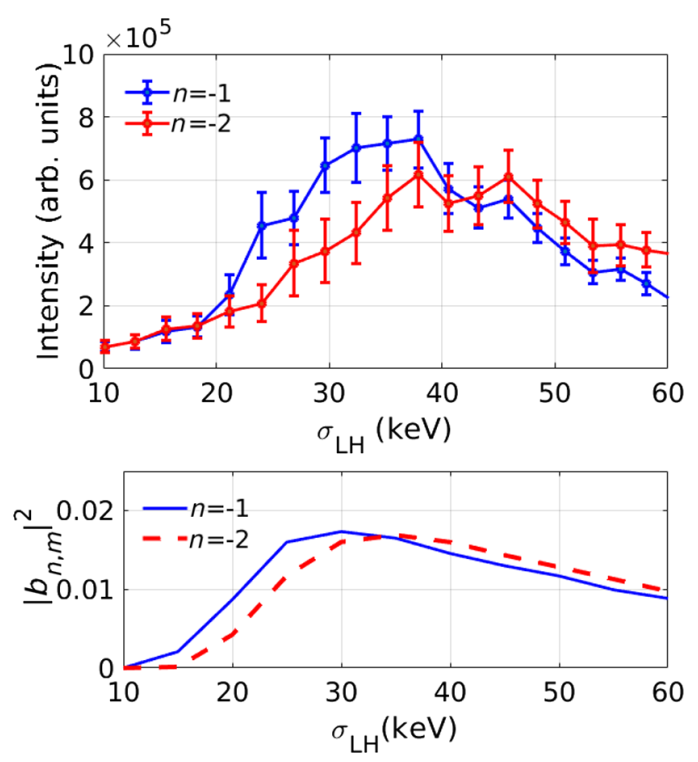

FIG. 4. Top: FEL intensity vs LH-induced energy spread. The FEL parameters are same as in Fig. 2. Bottom: square bunching factor. The calculated value of $p_{1}\left(\lambda_{\mu}\right)$ and $p_{2}\left(\lambda_{\mu}\right)$ in Fig. 2 are used to evaluate bunching factor.

The figure shows that, once the FEL emission is built up for the seed laser pulse energy of $\sim 15-20 \mu \mathrm{J}$, the intensity is weakly affected by even larger seed energies. In particular, a stronger seed laser is not able to recover the intensity reduction due to a weaker heating effect. The right plots provide the theoretical explanation of the experimental data. They show the spectrum of broadband energy modulation at the exit of the second modulator for different coherent energy modulations from the first seed laser, at two LH pulse energies. The contour plots are generated by inserting the energy distribution modified by the first seed laser into Eq. (15), which allows us to calculate the MBI gain at the exit of the first DS. Using such spectral gain function as an input to Eq. (13), the spectrum of the MBIinduced broadband energy modulation at the exit of the second modulator is eventually derived as function of the first seed pulse energy. An increase of the first coherent modulation is effective in removing incoherent energy modulations at initial wavelengths shorter than $10 \mu \mathrm{m}$, or $\sim 1 \mu \mathrm{m}$ at the entrance of the undulator. However, the effect becomes negligible immediately at longer wavelengths.

We plugged these two sets of energy modulation and different first seed energies into Eqs. (3) and (12), thus calculated the bunching factor $\left(b_{-2,20}\right)$ and the product function $(\Gamma)$, see bottom plots. It is shown that, while a strong beam heating is able to shift the product function to 1 or so, an increase of $A_{1}$ is not able to recover a unitary product function (compare red stars and blue stars for $\sigma_{\mathrm{LH}}=16 \mathrm{keV}$ and $\sigma_{\mathrm{LH}}=24 \mathrm{keV}$, respectively). The second plot of the second row compares the bunching factor with [dashed lines with stars, Eq. (13)] and without MBI 

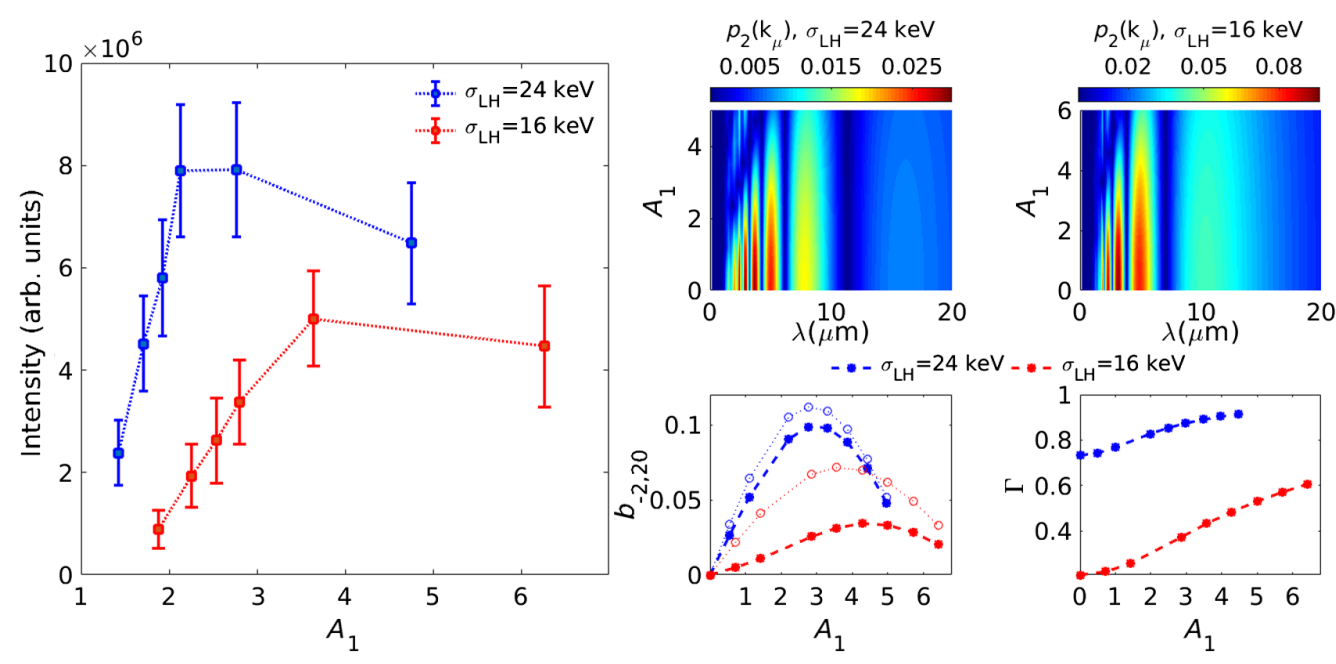

FIG. 5. Left: FEL intensity vs first seed laser pulse energy. Blue and red lines are experimental data for LH-induced energy spread $\sigma_{\mathrm{LH}}=24 \mathrm{keV}$ and $\sigma_{\mathrm{LH}}=16 \mathrm{keV}$. Right-top row: beam energy modulation amplitude as function of the (compressed) MBI modulation wavelength and first seed coherent energy modulation amplitude (seed energy), at the exit of the second modulator. Right-bottom row: bunching factor and product function vs first seed coherent energy modulation amplitude. Dotted lines with circles show $\dot{b}_{-2,20}$, dashed lines with stars show $b_{-2,20}$ in Eq. (11). The two subplots refer to the LH inducing $24 \mathrm{keV}$ and $16 \mathrm{keV}$ rms energy spread, respectively.

[doted lines with circle, Eq. (3)]. The plot illustrates the degradation of the bunching factor by MBI, at different coherent energy modulation amplitudes from the first seed. Finally, we find that the measured FEL intensity (left plot) at the two LH pulse energies is in agreement with the theoretical behavior of the bunching factor: by increasing the first seed energy, the FEL intensity grows up, to eventually fall down for excessive seeding energies.

\section{CONCLUSIONS}

Electron beam imperfections play a significant role in determining the spectral bandwidth and the pulse intensity of EEHG FEL emission. While EEHG is predicted to be more robust than other external seeding schemes to energy distortions that occur upstream, it is also anticipated that distortions that occur between the EEHG chicanes can significantly impact the FEL spectrum. This due in part to the combination of a strong magnetic chicane in the EEHG setup (compared to HGHG, for example) and LSC forces acting through the second modulator that together can amplify incoherent microbunching generated upstream in the accelerator. Here, the role played by the instability in different EEHG configurations was illustrated with experimental data, and good agreement was found with start-toend semianalytical results for the perturbed bunching factor. As such, the model turns out to be a practical tool for the design and optimization of short wavelength EEHG sources. Moreover, different balances of the strength of the two DSs change the impact of MBI onto the FEL spectrum and intensity, with smaller value of the $|n|$-factor less sensitive to incoherent energy modulations, as predicted. Finally, attempts to maximize the FEL intensity with a stronger coherent energy modulation from the first seed laser pulse were successful only for moderate or strong beam heating in the first stages of acceleration. This identifies a limitation in recovering optimal EEHG performance through the seed laser pulse energy. Further, it suggests a careful control of the instability in the accelerator, and a consequent optimization of the EEHG setup in the presence of relatively large heating levels.

\section{APPENDIX}

The derivation of Eq. (13) follows the strategy proposed in Ref. [21]. Starting from the general expression (4) in presence of energy distortions, we retain only the lowest order contribution near the harmonic,

$$
\begin{aligned}
b(k) \approx & \int_{-\infty}^{+\infty} d z \chi(z, k) e^{i\left(k-k_{E}+n \psi_{1}+m \psi_{2}\right) z} \\
& \times e^{i\left(-\zeta_{E} \Delta p_{1}(z)-a_{E} B_{2} \Delta p_{2}\left(z_{1}\right)\right)} .
\end{aligned}
$$

From here on, we will assume that $\Delta p_{1}$ is small enough not to alter significantly the phase space distribution after the first dispersive region. Also, we will further simplify our calculation taking $\Delta p_{2}\left(z_{1}\right)=\Delta p_{2}(z)$. This assumption is true as long as $\Delta p_{2}\left(z_{1}\right)$ is a sufficiently slowly varying function.

Using the definition of $\Delta p_{1,2}$ given in Eq. (1), and the identity is

$$
e^{i x \sin \theta}=\sum_{n=-\infty}^{\infty} J_{n}(x) e^{i n \theta}
$$


It is possible to expand the phase associated to broadband energy modulations,

$$
\begin{aligned}
e^{\left[-i \zeta_{E} \Delta p_{1}(z)\right]}= & \prod_{\mu=0}^{\infty} \sum_{l_{1}=-\infty}^{\infty} \exp \left[i l_{1} k_{\mu} z+i l_{1} \phi_{1 \mu}\right] \\
& \times J_{l_{1}}\left[-\zeta_{E} p_{1}\left(k_{\mu}\right)\right], \\
e^{\left[-i a_{E} B_{2} \Delta p_{2}(z)\right]}= & \prod_{\mu=0}^{\infty} \sum_{l_{2}=-\infty}^{\infty} \exp \left[i l_{2} k_{\mu} z+i l_{2} \phi_{2 \mu}\right] \\
& \times J_{l_{2}}\left[-a_{E} B_{2} p_{2}\left(k_{\mu}\right)\right],
\end{aligned}
$$

and Eq. (A1) reduces to Eq. (9).

The maximum EEHG bunching amplitude evaluated for $k=a_{E} k_{1 s}$ is

$$
b_{n, m}\left(a_{E} k_{1 s}\right)=\grave{b}_{n, m} \prod_{\mu=0}^{\infty} J_{0}\left(-\zeta_{E} p_{1}\left(k_{\mu}\right)\right) J_{0}\left(-a_{E} B_{2} p_{2}\left(k_{\mu}\right)\right) .
$$

Assuming that the energy distortions amplitudes, namely $p_{1,2}$, are small compared to the energy modulations induced by the seed laser, we can expand the Bessel functions around 0 . For a generic $\alpha$, we have

$$
J_{\alpha}(x)=\sum_{m=0}^{\infty} \frac{(-1)^{m}}{m ! \Gamma(m+\alpha+1)}\left(\frac{x}{2}\right)^{2 m+\alpha} .
$$

Setting $\alpha$ equal to zero, we truncate the series at second order in distortion amplitudes, obtaining

$$
\begin{aligned}
b_{n, m}\left(a_{E} k_{1 s}\right) \approx & \grave{b}_{n, m} \prod_{\mu=0}^{\infty}\left[1-\frac{1}{4}\left(\left(\zeta_{E} p_{1}\left(k_{\mu}\right)\right)^{2}\right.\right. \\
& \left.\left.+\left(a_{E} B_{2} p_{2}\left(k_{\mu}\right)\right)^{2}\right)\right]+\mathcal{O}\left(p_{1}^{3}, p_{2}^{3}\right) .
\end{aligned}
$$

[1] S. V. Milton et al., Exponential gain and saturation of a self-amplified spontaneous emission free-electron laser, Science 292, 2037 (2001).

[2] W. Ackermann et al., Operation of a free-electron laser from the extreme ultraviolet to the water window, Nat. Photonics 1, 336 (2007).

[3] P. Emma et al., First lasing and operation of an angstromwavelength free-electron laser, Nat. Photonics 4, 641 (2010).

[4] E. Allaria et al., Highly coherent and stable pulses from the fermi seeded free-electron laser in the extreme ultraviolet, Nat. Photonics 6, 699 (2012).

[5] E. Allaria et al., Two-stage seeded soft-x-ray free-electron laser, Nat. Photonics 7, 913 (2013).
[6] G. Stupakov, Using the Beam-Echo Effect for Generation of Short-Wavelength Radiation, Phys. Rev. Lett. 102, 074801 (2009).

[7] D. Xiang and G. Stupakov, Echo-enabled harmonic generation free electron laser, Phys. Rev. ST Accel. Beams 12, 030702 (2009).

[8] E. Hemsing, M. Dunning, C. Hast, T. O. Raubenheimer, S. Weathersby, and D. Xiang, Highly coherent vacuum ultraviolet radiation at the 15 th harmonic with echo-enabled harmonic generation technique, Phys. Rev. ST Accel. Beams 17, 070702 (2014).

[9] E. Hemsing, M. Dunning, B. Garcia, C. Hast, T. Raubenheimer, G. Stupakov, and D. Xiang, Echo-enabled harmonic generation has been used to seed a free-electron laser and has been demonstrated up to the 75th harmonic, producing $32 \mathrm{~nm}$ light from a 2,400 nm laser, Nat. Photonics 10, 512 (2016).

[10] P. R. Ribic et al., Coherent soft x-ray pulses from an echo-enabled harmonic generation free-electron laser, Nat. Photonics 7, 1749 (2019).

[11] C. Feng et al., Coherent extreme ultraviolet free-electron laser with echo-enabled harmonic generation, Phys. Rev. Accel. Beams 22, 050703 (2019).

[12] N. S. Mirian et al., Spectrotemporal control of soft x-ray laser pulses, Phys. Rev. Accel. Beams 23, 060701 (2020).

[13] G. Stupakov, Effect of finite pulse length and laser frequency chirp on HGHG and EEHG seeding, Technical Report No. SLAC-PUB-14639, 2011.

[14] D. Ratner, A. Fry, G. Stupakov, and W. White, Laser phase errors in seeded free electron lasers, Phys. Rev. ST Accel. Beams 15, 030702 (2012).

[15] E. Hemsing, Minimum spectral bandwidth in echo seeded free electron lasers, Front. Phys. 7, 35 (2019).

[16] Z. Huang, D. Ratner, G. Stupakov, and D. Xiang, Effects of energy chirp on echo-enabled harmonic generation freeelectron lasers, in Proceedings of the 2009 Free-Electron Laser Conference (2009), p. 127.

[17] G. Penn, Stable, coherent free-electron laser pulses using echo-enabled harmonic generation, Phys. Rev. ST Accel. Beams 17, 110707 (2014).

[18] P. R. Ribič, E. Roussel, G. Penn, Gi. De Ninno, L. Giannessi, G. Penco, and E. Allaria, Echo-enabled harmonic generation studies for the Fermi free-electron laser, Photonics 4, 19 (2017).

[19] G. Penn, EEHG performance and scaling laws, NGLS Technical Note 35, LBNL, 2012.

[20] E. Hemsing, B. Garcia, Z. Huang, T. Raubenheimer, and D. Xiang, Sensitivity of echo enabled harmonic generation to sinusoidal electron beam energy structure, Phys. Rev. Accel. Beams 20, 060702 (2017).

[21] E. Hemsing, Bunching phase and constraints on echo enabled harmonic generation, Phys. Rev. Accel. Beams 21, 050702 (2018).

[22] Z. Zhang, R. Lindberg, W. M. Fawley, Z. Huang, J. Krzywinski, A. Lutman, G. Marcus, and A. Marinelli, Microbunching-instability-induced sidebands in a seeded free-electron laser, Phys. Rev. Accel. Beams 19, 050701 (2016).

[23] K. Zhang, L. Zeng, Z. Qi, C. Feng, and D. Wang, Eliminating the microbunching-instability-induced 
sideband in a soft $\mathrm{x}$-ray self-seeding free-electron laser, Nucl. Instrum. Methods Phys. Res., Sect. A 882, 22 (2018).

[24] G Perosa et al., Linear optics control of sideband instability for improved free-electron laser spectral brightness, Phys. Rev. Accel. Beams 23, 110703 (2020).

[25] E. Hemsing et al., Soft x-ray seeding studies for the slac linac coherent light source ii, Phys. Rev. Accel. Beams 22, 110701 (2019).

[26] Z. Huang and K. J. Kim, Formulas for coherent synchrotron radiation microbunching in a bunch compressor chicane, Phys. Rev. ST Accel. Beams 5, 074401 (2002).

[27] E. L. Saldin, E. A. Schneidmiller, and M. V. Yurkov, Klystron instability of a relativistic electron beam in a bunch compressor, Nucl. Instrum. Methods Phys. Res., Sect. A 490, 1 (2002).

[28] S. Heifets, G. Stupakov, and S. Krinsky, Coherent synchrotron radiation instability in a bunch compressor, Phys. Rev. ST Accel. Beams 5, 064401 (2002).

[29] D. Ratner, C. Behrens, Y. Ding, Z. Huang, A. Marinelli, T. Maxwell, and F. Zhou, Time-resolved imaging of the microbunching instability and energy spread at the linac coherent light source, Phys. Rev. ST Accel. Beams 18, 030704 (2015).
[30] Z. Huang et al., Measurements of the linac coherent light source laser heater and its impact on the x-ray free-electron laser performance, Phys. Rev. ST Accel. Beams 13, 020703 (2010).

[31] Z. Huang, M. Borland, P. Emma, J. Wu, C. Limborg, G. Stupakov, and J. Welch, Suppression of microbunching instability in the linac coherent light source, Phys. Rev. ST Accel. Beams 7, 074401 (2004).

[32] S. Di Mitri, G. Perosa et al., Experimental evidence of intrabeam scattering in a free-electron laser driver, New J. Phys. 22, 083053 (2020).

[33] G. Perosa and S. Di Mitri, Matrix model for collective phenomena in electron beam's longitudinal phase space, Sci. Rep. 11, 7895 (2021).

[34] E. L Saldin, E. A Schneidmiller, and M. V. Yurkov, Longitudinal space charge-driven microbunching instability in the tesla test facility linac, Nucl. Instrum. Methods Phys. Res., Sect. A 528, 355 (2004).

[35] J. Lee, J.-H. Han, S. Lee, J. Hong, C. H.Kim, C. K. Min, and I. S. Ko, PAL-XFEL laser heater commissioning, Nucl. Instrum. Methods Phys. Res., Sect. A 843, 39 (2017).

[36] S. Spampinati et al., Laser heater commissioning at an externally seeded free-electron laser, Phys. Rev. ST Accel. Beams 17, 120705 (2014). 